\title{
Free Triiodothyronine Measurement
}

National Cancer Institute

\section{Source}

National Cancer Institute. Free Triiodothyronine Measurement. NCI Thesaurus. Code C74787.

The determination of the amount of free triiodothyronine present in a sample. 